\title{
A study of Integrated Circuit Dicing Tape when used in a Plasma Dicing Environment (Sept 2019)
}

\author{
Harry Fulton, Oliver Ansell, Janet Hopkins, Martin Hanicinec, Takuo Nishida and Taku Umemoto, \\ Lijie Li, Member, IEEE
}

\begin{abstract}
The main objective of this study was to establish which of the many dicing tapes used in the semiconductor industry, would be most suitable for use in plasma dicing. Tape design over the past 40 years has continually evolved through advancements in both dicing technologies and the incessant revision of integrated circuit packaging. Die singulation has traditionally been accomplished using a diamond saw, laser-based technology or combinations of both. The stress on dicing tape was therefore limited to fatigue through the physical nature of saw dicing or heat energies induced during laser dicing. These processes do not expose dicing tape to either high vacuum or a variety of plasma chemistry. This investigative work is a continuation of studies examining dicing tape behavior when employed for use in plasma dicing. Results show that Polyolefin (PO) UV-tape with low to medium adhesion strength exhibit the greatest resilience in harsh plasma etch conditions and that efficient photoinitiated cross-linking (or curing) of the adhesive is triggered if directly exposed to the photonic energies present in a pure SF6 plasma or full plasma dicing process. However, the post plasma dice, post UV cure adhesive strength can still be minimized if the tape manufacturers recommended time limitations between tape mount, plasma dice and the die pick-up process are adhered to.
\end{abstract}

Index Terms - dicing, plasma, singulation, UV-tape, anti-ESD

\section{INTRODUCTION}

The challenges to manufacture smaller, substantially thinner and stronger integrated circuits has encouraged many chip manufacturers to consider alternative die singulation techniques. Some have already moved away from traditional saw dicing to various laser-based technologies and others are evaluating or have already made a strategic switch to a plasma dicing approach. The non-aggressive chemical elimination of dicing lane material using a Bosch process, leverages some distinct advantages over the more physical methods of device singulation [1-3]. The parallel nature of this process can yield significant throughput benefits, especially with smaller and thinner devices. However, the increase in die per wafer from supporting dicing lanes as narrow as $5 \mu \mathrm{m}$, are also of dividend. With higher die strengths achievable using plasma dicing, the reliability essential in today's flexible electronics and multi-stacked devices, makes plasma dicing technology even more appealing $[4,5]$.

Most of the development of new dicing tapes for use in a myriad of various saw blade techniques and the more recent laser dicing or stealth dicing methods, has come from Japan. Tape performance is well documented and very often designed for use in a specific application [6-9]. e.g. LINTEC's Adwill D-821 HS tape was designed with a specific laser transmittance to facilitate

This work was supported by SPTS Technologies, LINTEC Advanced Technologies (Europe) GmbH and Swansea University. H. Fulton, O. Ansell and J. Hopkins are with the Department of Engineering, SPTS Technologies Ltd., Newport NP18 2TA, U.K. (e-mail: stewart.fulton@ spts.com).

Martin Hanicinec is currently studying for his doctorate at UCL (University College London).
Takuo Nishida and Taku Umemoto are with LINTEC Advanced Technologies (Europe) GmbH, Konrad-Zuse-Platz 1, 81829 München, Germany

Lijie Li is with the College of Engineering, Swansea University, Swansea SA2 8PP, U.K. (e-mail: 1.li@ swansea.ac.uk). 
Stealth Dicing through tape [9]. Tape selection depends not only on the dicing technology being used but also on die size and thickness. With this knowledge, a suitable tape is selected to ensure each die is held securely during the dicing process, yet still permit the individual die to be reliably 'picked' from the tape prior to further processing or packaging.

The main objective of this study was to establish which of the many dicing tapes currently available, would be most suitable for use in plasma dicing. Fig. 1 shows how fully processed wafers are frame mounted on dicing tape in preparation for plasma dicing into singulated individual die.

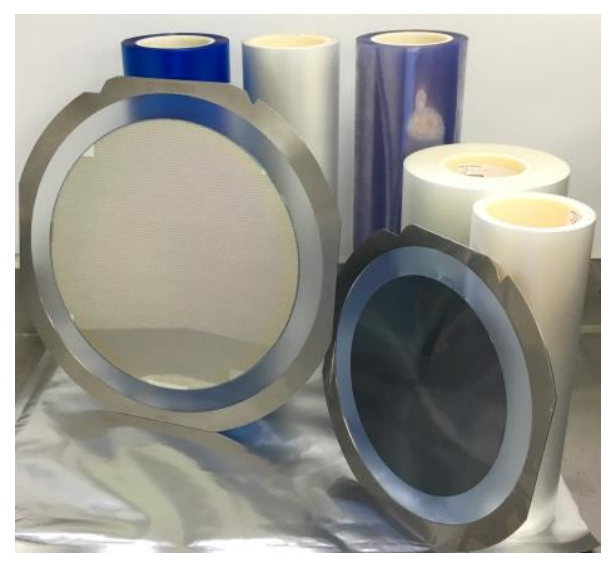

Fig. 1. Processed semiconductor wafers frame mounted on dicing tape

The plasma dicing system utilized in this research was an SPTS 300mm Rapier ${ }^{\mathrm{TM}} \mathrm{S}$ module attached to an SPTS Mosaic ${ }^{\mathrm{TM}}$ handler. The module is specifically designed to remove the silicon in the dicing lanes between semiconductor devices while mounted on dicing tape. This is achieved using a time multiplexed deep anisotropic etching technique more commonly known as the Bosch Process [10]. The tape mounted wafer is cooled using an ESC (electrostatic chuck) during the entire process [11].

The Bosch process is still the industry standard for deep silicon etching and is extensively used in MEMS and TSV applications $[12,13]$.

In addition to the fluorinated gases; $\mathrm{SF}_{6}$ (Sulphur Hexafluoride) and $\mathrm{C}_{4} \mathrm{~F}_{8}$ (Octafluorocyclobutane) used in the Bosch Process, dicing tape is also exposed to Argon and Oxygen plasma during a typical plasma dicing process. An Argon plasma is required for effective clamping and declamping of the substrate on the monopolar ESC. $\mathrm{O}_{2}$ can be used instead of $\mathrm{SF}_{6}$ in the polymer removal step and is frequently used post plasma dicing to remove the passivation polymer deposited on the device sidewalls or for an ashing (or strip) process for photo resist removal. If a dielectric etch is required to remove a thin oxide layer covering the silicon dicing lane prior to dicing, $\mathrm{O}_{2}$ and Ar may be part of the gas mixture required for this etch.

Fig. 2 shows a simplified illustration of the Rapier S plasma dicing module. 


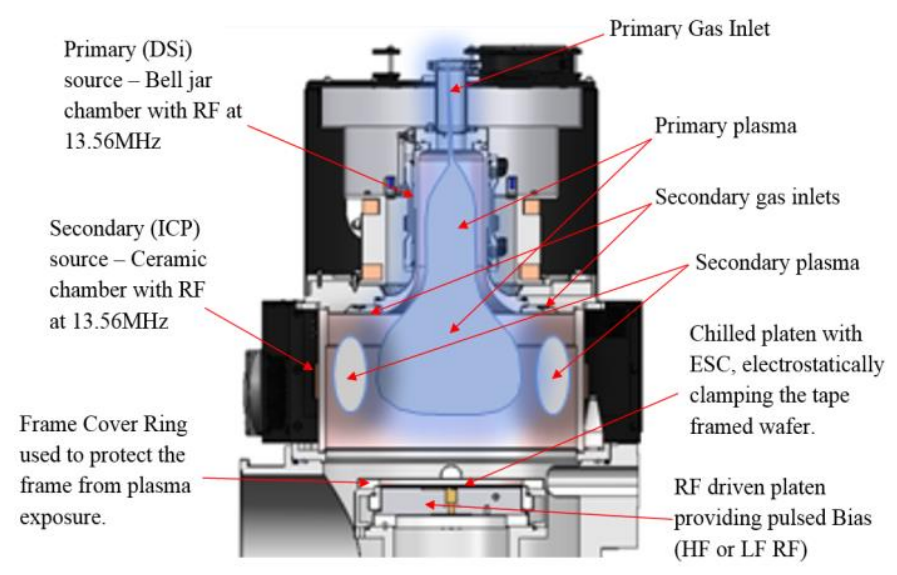

Fig. 2. SPTS Rapier 200S Plasma Dicing Module

\section{MEASUREMENT EQUIPMENT AND SAMPLE PREPARATION}

In most cases, sample preparation and test procedures were aligned with specifications detailed in the Japanese Industrial Standard document JIS Z 0237 - 'Testing methods of pressure-sensitive adhesive tapes and sheets' [14] or the equivalent ISO standards $[15,16]$. However, wafer size or the limitations of the measurement apparatus necessitated some minor variations to these tests. Table 1. lists the different test apparatus utilized during this research.

Table 1. Test apparatus used during the research

\begin{tabular}{|c|c|c|}
\hline $\begin{array}{c}\text { Test } \\
\text { Equipment }\end{array}$ & Model details & Further information \\
\hline Wafer Mounter & $\begin{array}{l}\text { ADT (Advanced } \\
\text { Dicing } \\
\text { Technologies) } \\
\text { Model } 966 \text { - }\end{array}$ & $\begin{array}{l}\text { Manual wafer mounting system, able to } \\
\text { handle } 300 \mathrm{~mm} \text { and } 400 \mathrm{~mm} \text { rolls of } \\
\text { dicing tape. } 296 \mathrm{~mm} \text { or } 396 \mathrm{~mm} \text { dicing } \\
\text { frames. }\end{array}$ \\
\hline $\begin{array}{c}\text { Tension \& } \\
\text { Compression } \\
\text { tester }\end{array}$ & $\begin{array}{c}\text { Mecmesin } \\
\text { Multitest 2.5dV }\end{array}$ & $\begin{array}{l}\text { Used during tape elongation; tensile } \\
\text { strength and adhesion peel strength } \\
\text { measurements - }\end{array}$ \\
\hline $\begin{array}{l}\text { UV irradiation } \\
\text { system }\end{array}$ & $\begin{array}{l}\text { Stag SE 100T } \\
\text { EPROM Eraser }\end{array}$ & $\begin{array}{l}\text { Calibrated against a LINTEC RAD } \\
2010 \text { using a mercury lamp with a } \\
\text { targeted wavelength of } 365 \mathrm{~nm} \text { at a UV } \\
\text { intensity of } 230 \mathrm{~mW} / \mathrm{cm}^{2} \text { and UV } \\
\text { dosage of } 190 \mathrm{~mJ} / \mathrm{cm}^{2}\end{array}$ \\
\hline $\begin{array}{l}\text { SEM (Scanning } \\
\text { Electron } \\
\text { Microscope) }\end{array}$ & Zeiss Sigma HV & $\begin{array}{l}\text { Samples sputter coated on the adhesive } \\
\text { side with gold to minimize charge } \\
\text { accumulation. Images captured at mags } \\
\text { of } 150 \mathrm{x}, 500 \mathrm{x} \& 2 \mathrm{kx} \text { at } 10 \mathrm{kV} \text { with a } \\
\text { working distance of } 5 \mathrm{~mm} \text {. }\end{array}$ \\
\hline $\begin{array}{l}\text { FTIR-ATR } \\
\text { (Fourier- } \\
\text { transform } \\
\text { infrared }\end{array}$ & $\begin{array}{l}\text { Perkin-Elmer } \\
\text { Spectrum } 100\end{array}$ & $\begin{array}{l}\text { Utilizing a diamond crystal to allow } \\
\text { measurement depths up to } 3 \mu \mathrm{m} \text {. }\end{array}$ \\
\hline
\end{tabular}

spectroscopy 
w/attenuated

total reflectance

accessory)

\section{TAPE ROBUSTNESS TEST}

As reported in the conference paper 'Dicing Tape Performance in a Plasma Dicing Environment' [17], all dicing tapes out-gas to varying degrees. This occurs as the volatile organic compounds (VOCs) within the base film \& adhesive diffuse, sublime or vaporize and release as gases. The outgassing rate is accelerated if materials are subjected to heat and vacuum conditions $[18,19]$.

If the tape outgassing rate is excessive within a plasma dicing reactor, the gas released can form small to large pockets between the tape and the substrate. This can prove problematic if the force exerted by gas built up in pockets exceeds that of the electrostatic gripping force between the electrostatic chuck (ESC) and tape mounted wafer. An example of excessive tape outgassing and the consequences are shown in fig. 3.

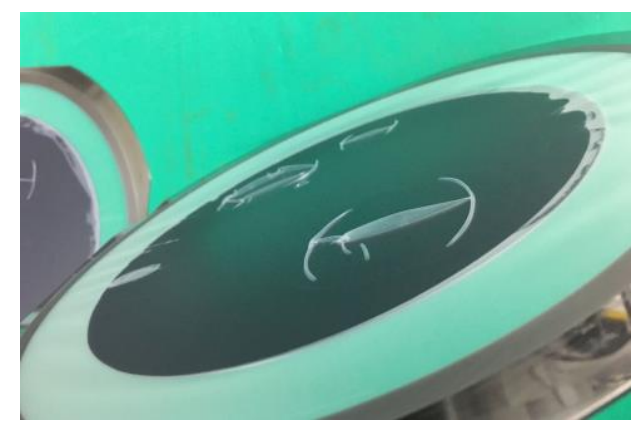

Fig. 3. An example of outgassing seen during 'tape robustness' tests. This sample shows evidence of collapsed gas blisters formed between the dicing tape and silicon substrate.

An initial set of tape robustness tests were conducted on 14 different LINTEC Adwill dicing tapes. This was supplemented through the testing of an additional 40 dicing tapes supplied by Nitto Denko Corporation, Furukawa Electric Group, Denka Company Limited, Ultron Systems Inc, D\&X Co. Ltd., Sekisui Chemical Co. Ltd. and some other tapes from LINTEC Corporation.

The same methodology used to measure the 'risk of declamp' on the preliminary set of LINTEC Adwill dicing tapes was adhered to. i.e. $200 \mathrm{~mm}$ bare silicon wafers were tape mounted on $296 \mathrm{~mm}$ metal frames and processed using an aggressive, non-switched, silicon etch process. Helium back-side wafer cooling, normally employed to act as a heat transfer medium during the standard plasma dicing process, was not used during the 'robustness test'. The recipe included an incremental reduction in the ESC clamping voltage over 18 separate 10 second steps while, continuously monitoring for substrate declamp using SPTS's patented Sentinel ${ }^{\text {TM }}$ technology [20].

The 50 or so dicing tapes tested included tapes with base films of polyolefin (PO), polyvinylchloride (PVC); with and without Di-2-ethylhexyl phthalate (DEHP) plasticizers, polyethylene terephthalate (PET), ethylene-vinyl acetate (EVA) and polyethylene naphthalate (PEN). All but one tape had acrylic adhesive layers. Most were UV tapes, but several non-UV tapes were tested. Eight 
of the tapes tested had anti-ESD properties, either within the base film, within the adhesive or as a thin conductive layer between the base film and adhesive or on the adhesive surface.

The recipe developed for this test was designed to encourage tape outgassing and allow a performance rating between those tested. Surface temperatures measured on the $200 \mathrm{~mm}$ silicon substrates mounted on tapes that survived the complete (18 step) process, were on average $75-80 \mathrm{degsC}$. Temperature dots covered in Kapton tape were used during these tests.

Fig. 4. shows the ESC clamping voltage stepping down in increments of 100v and the Sentinel signal from a full 18 step process where no declamp was experienced.

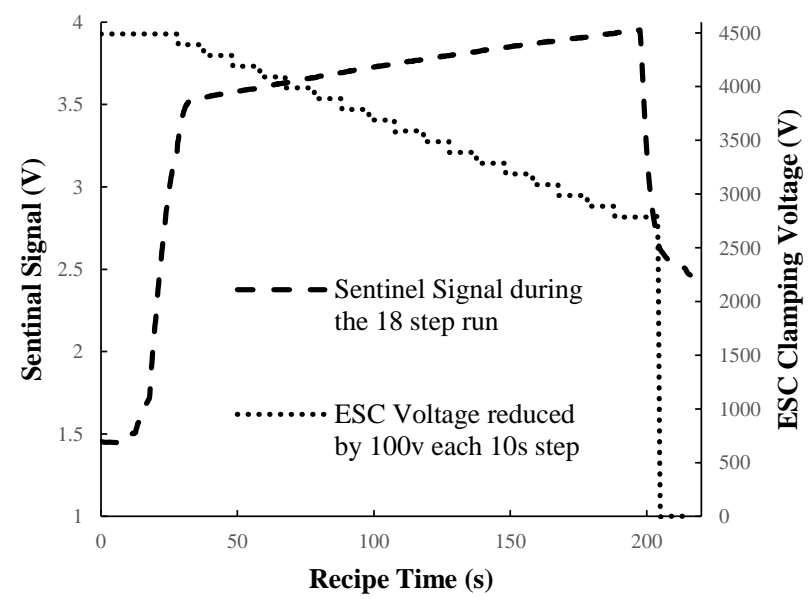

Fig. 4. Trace showing the Sentinel ${ }^{\mathrm{TM}}$ signal and the ESC clamping voltage during entire declamp test recipe.

Fig. 5 shows the Sentinel ${ }^{\mathrm{TM}}$ detecting a declamp event on step 11 of the STD_DeclampTest recipe run on tape A3.

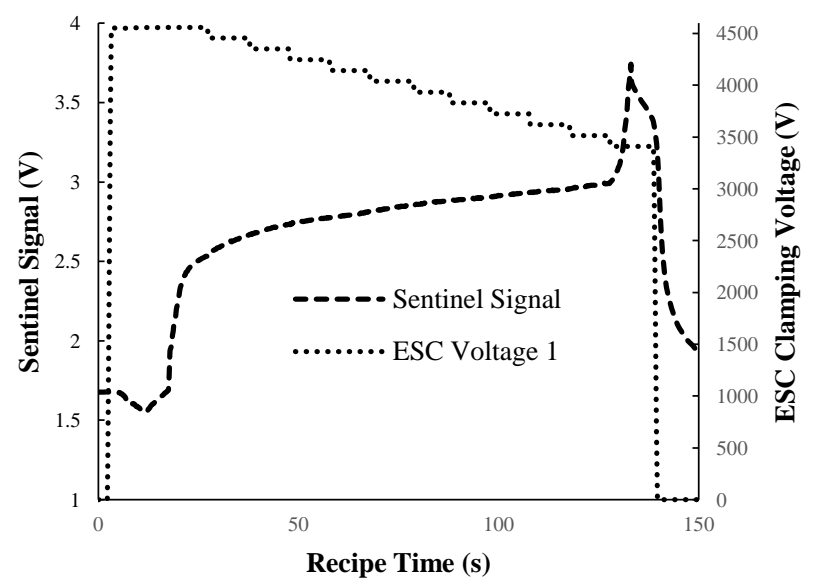

Fig. 5. Sentinel ${ }^{\mathrm{TM}}$ detects declamp during step 11 of declamp test recipe as seen by the sharp increase in the Sentinel ${ }^{\mathrm{TM}}$ signal. $^{2}$

Table 2 shows a sample of results from all the tapes evaluated so far using the 'robustness test'. Base film and adhesive properties 
were taken from the tape data sheets.

Table 2. A sample of 'tape robustness test' results from the 50+ dicing tapes tested so far. The illustration includes tapes from all 7 Tape Manufacturers (coded A to $\mathrm{G})$, showing the variation in 'risk of declamp' during plasma dicing.

\begin{tabular}{|c|c|c|c|c|c|c|}
\hline $\begin{array}{c}\text { Tape } \\
\text { Supplier }\end{array}$ & $\begin{array}{c}\text { Tape } \\
\text { ID }\end{array}$ & $\begin{array}{c}\text { Base Film } \\
\text { \& Adhesive } \\
\text { Type }\end{array}$ & $\begin{array}{c}\text { Base \& } \\
\text { Adhesive } \\
\text { THK }\end{array}$ & $\begin{array}{c}\text { Adhesive } \\
\text { Strength } \\
\text { (pre/post } \\
\text { UV cure) } \\
\text { [mN/25m } \\
\text { m] }\end{array}$ & $\begin{array}{l}\text { Avg. } \\
\text { Score }\end{array}$ & $\begin{array}{c}\text { Risk of } \\
\text { Declamp }\end{array}$ \\
\hline A & A10 & PO UV & $80 / 5$ & $4900 / 80$ & 19.0 & Very Low \\
\hline A & A9 & PO UV & $80 / 5$ & $2800 / 160$ & 19.0 & Very Low \\
\hline $\mathrm{A}$ & A11 & PO UV & $80 / 10$ & $3200 / 150$ & 19.0 & Very Low \\
\hline B & $\mathrm{B} 1$ & PO UV & $100 / 10$ & $1200 / 100$ & 19.0 & Very Low \\
\hline $\mathrm{D}$ & D1 & PO-ESD UV & $80 / 18$ & $2942 / 245$ & 18.3 & Very Low \\
\hline $\mathrm{C}$ & $\mathrm{C} 1$ & PO UV & $80 / 8$ & $2750 / 125$ & 18.0 & Very Low \\
\hline B & B2 & PO UV & $80 / 5$ & $1100 / 200$ & 17.7 & Low \\
\hline G & G1 & PEN UV & $25 / 40$ & $200 / 100$ & 14.8 & Medium \\
\hline $\mathrm{C}$ & $\mathrm{C} 7$ & PET UV & $150 / 20$ & $\begin{array}{c}22500 / 12 \\
5\end{array}$ & 13.4 & Medium \\
\hline A & A 2 & PO UV & $80 / 15$ & $\begin{array}{c}12300 / 69 \\
0\end{array}$ & 13.0 & Medium \\
\hline $\mathrm{C}$ & $\mathrm{C} 8$ & $\begin{array}{c}\text { PET Non- } \\
\text { UV }\end{array}$ & $51 / 19$ & $425 / 425$ & 13.0 & Medium \\
\hline A & $\mathrm{A} 3$ & PO UV & $80 / 5$ & $8400 / 750$ & 12.8 & Medium \\
\hline B & B2 & PO UV & $80 / 5$ & $1100 / 200$ & 12.2 & Medium \\
\hline A & $\mathrm{A} 2$ & PO UV & $80 / 15$ & $\begin{array}{c}12300 / 69 \\
0\end{array}$ & 11.5 & Medium \\
\hline E & E4 & PO Non-UV & $100 / 30$ & $\begin{array}{c}1600 / 160 \\
0\end{array}$ & 11.2 & Medium \\
\hline
\end{tabular}




\begin{tabular}{|c|c|c|c|c|c|c|}
\hline $\mathrm{C}$ & $\mathrm{C} 3$ & PVC UV & $80 / 8$ & $6250 / 125$ & 4.4 & High \\
\hline $\mathrm{C}$ & $\mathrm{C} 5$ & $\begin{array}{c}\text { PVC Non- } \\
\text { UV }\end{array}$ & $65 / 10$ & $\begin{array}{c}1000 / 100 \\
0\end{array}$ & 3.8 & High \\
\hline $\mathrm{F}$ & $\mathrm{F} 1$ & PO-ESD UV & $80 / 5$ & $4263 / 138$ & 3.8 & High \\
\hline A & A14 & $\begin{array}{c}\text { PVC-DEHP } \\
\text { Free UV }\end{array}$ & $80 / 10$ & $3300 / 320$ & 3.0 & High \\
\hline A & A13 & $\begin{array}{c}\text { PVC-DEHP } \\
\text { UV }\end{array}$ & $80 / 10$ & $2800 / 290$ & 2.7 & High \\
\hline $\mathrm{C}$ & $\mathrm{C} 4$ & $\begin{array}{c}\text { PO -ESD } \\
\text { UV }\end{array}$ & $80 / 5$ & $5625 / 125$ & 2.6 & High \\
\hline & 14 & PO ESD UV & $80 / 5$ & $2250 / 125$ & 2.5 & High \\
\hline
\end{tabular}

Results from the additional tapes tested generally agreed with those from the initial LINTEC sample set, however not in all cases. PO tapes, with a hard adhesive (low to medium pre-UV adhesive strength) exhibited the greatest resilience and in most cases out-performed the PO tapes with a softer adhesive (high pre-UV adhesive strength). PVC and EVA tapes performed less well than PET or PEN tapes. Adhesive and base film thickness was determined not to be a major contributor to out-gassing rate but the inclusion of anti-ESD properties often increased the risk of declamp but again not in all cases. The most significant finding in testing a much larger range of tapes is that although the robustness test is useful as a measurement of the thermal conductance of tape and its propensity to outgas VOC's, there are broad differences in tape chemistry of the base film and adhesive between tape manufacturers. Further analysis of tape outgassing behaviours, possibly using Gas Chromatography-Mass Spectrometry would provide a greater understanding and aid in advancing this research.

Note that the remainder of tests detailed in this paper are on LINTEC Adwill tapes coded PO2, PO6, PO10 and PVC1. These are Lintec's high usage dicing tapes. P02 has a polyolefin base film and is UV curable tape typically used for small die $\left(1 \mathrm{~mm}^{2}\right.$ or less) were a high pre-UV adhesive strength is required to prevent die fly-off during the more mechanical dicing techniques. PO6 is a polyolefin non-UV tape of low to medium adhesive strength often employed in saw dicing of medium die $\left(>1<5 \mathrm{~mm}^{2}\right)$. PO10 is polyolefin UV curable tape with medium pre-UV adhesion strength and very low post UV adhesion strength. It can be used for various dicing techniques for medium size die where low pick-up force is required e.g. on more fragile thinner devices. Note that the base film properties of PO2, PO6 and PO10 are identical. They differ only in their adhesive layer properties. The last tape discussed is PVC1. It is Lintec's standard UV curable PVC (Polyvinyl Chloride) tape with a low to medium pre-UV adhesive strength, most often used for saw dicing of small die.

\section{ThE TAPE ELONGATION ANOMALY AFTER PLASMA EXPOSURE}

During the elongation and tensile strength testing of two polyolefin (PO) tapes and one PVC tape, a distinctive bump on the elongation curves was noted, as illustrated in a stacked representation of the cross-direction samples depicted in Fig 6. 


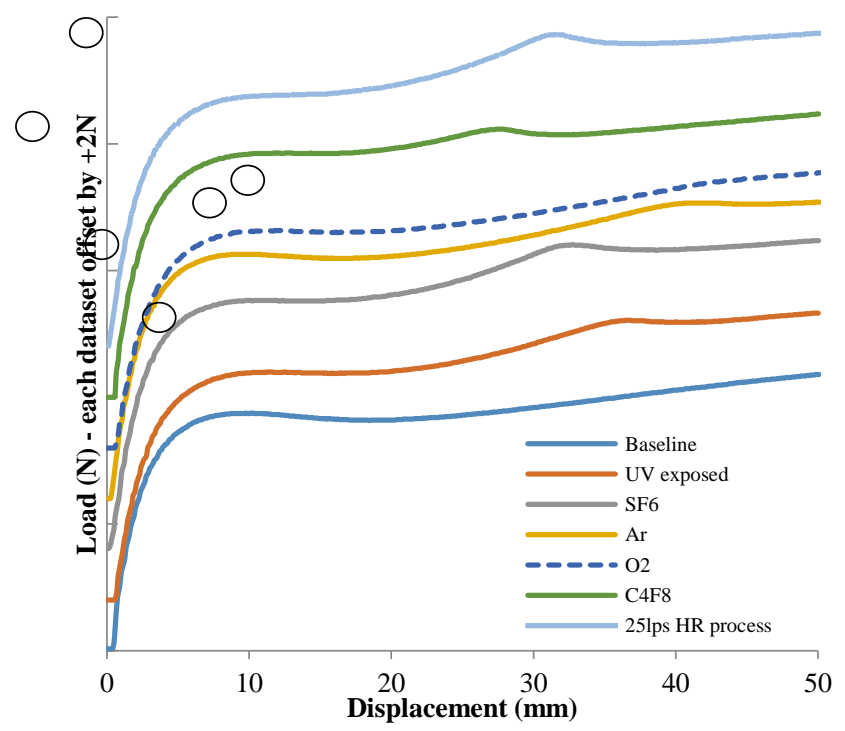

Fig. 6. Stacked representation of first 50mm of the PO10 tape elongation traces of cross direction after exposure to different plasma species

In Fig. 6, the Load data from the first $50 \mathrm{~mm}$ of each elongation curve has been deliberately offset by $+2.0 \mathrm{~N}$ cumulatively in order to illustrate the displacement position of each bump. This anomaly marked with a circle, has been shown to be the point at which the adhesive separates into bands on the surface of the base film. It occurs at different displacement on the samples either UV exposed in a UV irradiation system or exposed to the various individual plasma species or a partial plasma dicing process.

SEM analysis indicates that as the adhesive thins it reaches a critical elastic yield point then separates into bands. It is not seen on stretched unexposed (baseline) PO10 tape as shown in the left image of Fig. 7 but is evident in the UV exposed sample shown in right image of Fig. 7.

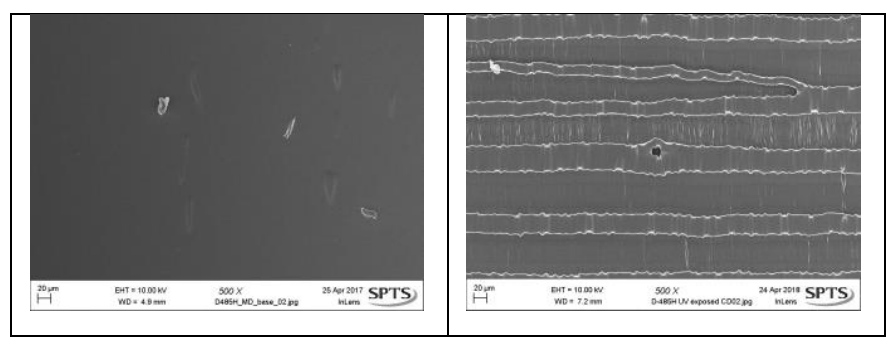

Fig. 7. Left - SEM image of stretched unexposed PO10 tape showing no evidence of adhesive banding. Right - SEM image of stretched PO10 UV irradiated in an EPPROM eraser.

Fig. 8 shows that the adhesive on PO10 exposed to 300 s of an $\mathrm{SF}_{6}$ plasma causes the adhesive to become firmly attached to the base film, reducing the base film's ability to stretch evenly, resulting in micro tears during the elongation test. 


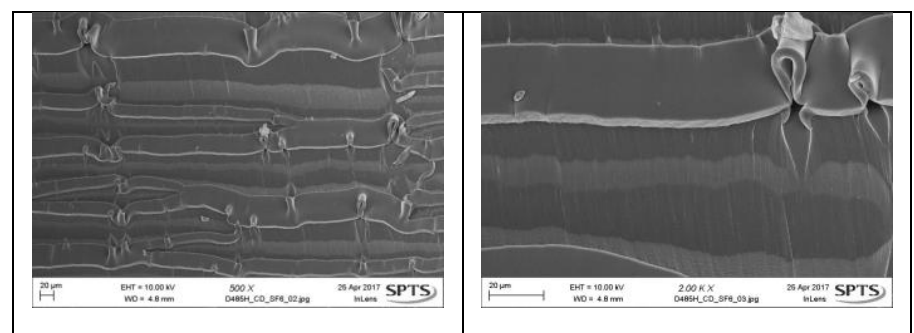

Fig. 8. SEM images of PO10 tape showing adhesive structure (after tape shear). Left to Right - after exposure to 300s of an SF6 plasma (500X), the same image at higher magnification showing micro tears caused by firmly attached adhesive (2000x)

Fig.9 shows that $\mathrm{PO} 10$ samples exposed to $\mathrm{Ar}, \mathrm{O}_{2}$ or $\mathrm{C}_{4} \mathrm{~F}_{8}$ plasma appear very different and that adhesive attachment to the base film is much lower, allowing the base film to stretch with far less inhibition.

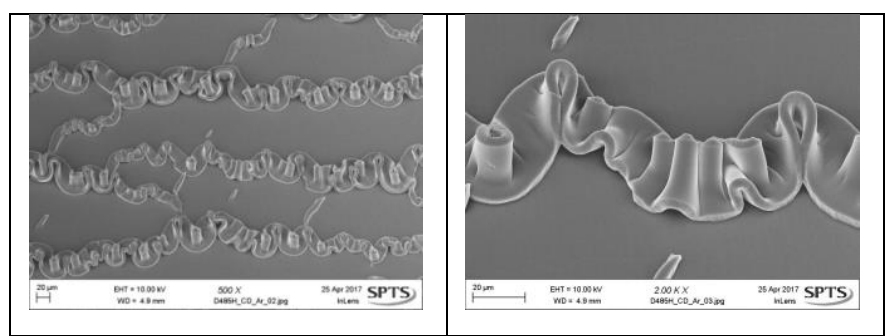

Fig. 9. SEM images of PO10 tape showing adhesive structure (after tape shear). Left to Right - after exposure to 300s of an Argon plasma $(500 \mathrm{X})$, the same image at higher magnification showing that the adhesive is not firmly attached to the base film.

The absence of adhesive banding on the stretched unexposed PO10 sample and its appearance on samples UV exposed or exposed to plasma within the plasma dicing module, suggested that the optical emissions spectra of certain plasmas can trigger the photo-initiated adhesive curing process otherwise known as adhesive cross-linking. The SEM analysis coupled with the elongation and tensile strength results reported in the conference paper 'Dicing Tape Performance in a Plasma Dicing Environment' [17] show that adhesive cross-linking occurs at different rates depending on the species of plasma used.

\section{Plasma EXPOSURE \& ADHESIVE CROSS-LINKING}

The results from the tape elongation and tensile strength tests prompted further investigation into a link between plasma exposure and adhesive curing. Samples were prepared in three steps as follows:

1. Frame mounting a $200 \mathrm{~mm}$ silicon wafer with the tape adhered to the polished side

2. Cutting the wafer from the initial mounting frame at the edge of the wafer

3. Remounting the $200 \mathrm{~mm}$ wafer with the taped polished side facing up and ready for plasma exposure as shown in Fig 10. 


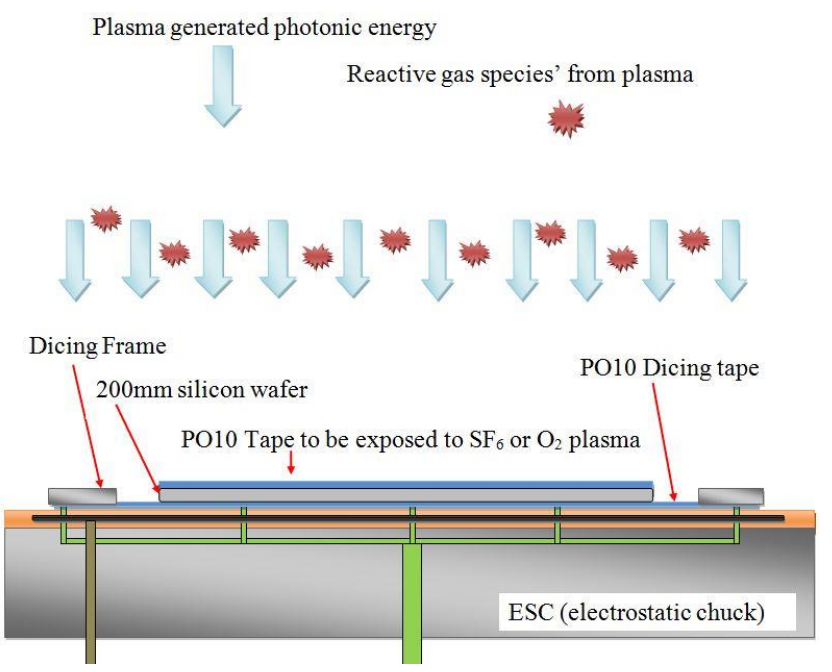

Fig. 10. Tape on polished silicon, then remounted on taped frame to provide a means of exposing the test tape to plasma [conference paper reference]

Each sample was then exposed to a predetermined $\mathrm{SF}_{6}$ or $\mathrm{O}_{2}$ plasma of equivalent intensity prior to measurement of adhesive peel strength.

The plasma exposure versus loss of adhesion peel strength results are detailed in Figure 11.

Findings demonstrate that plasma exposure can trigger the adhesive curing process and that the curing rate is dependent on plasma species and exposure time.

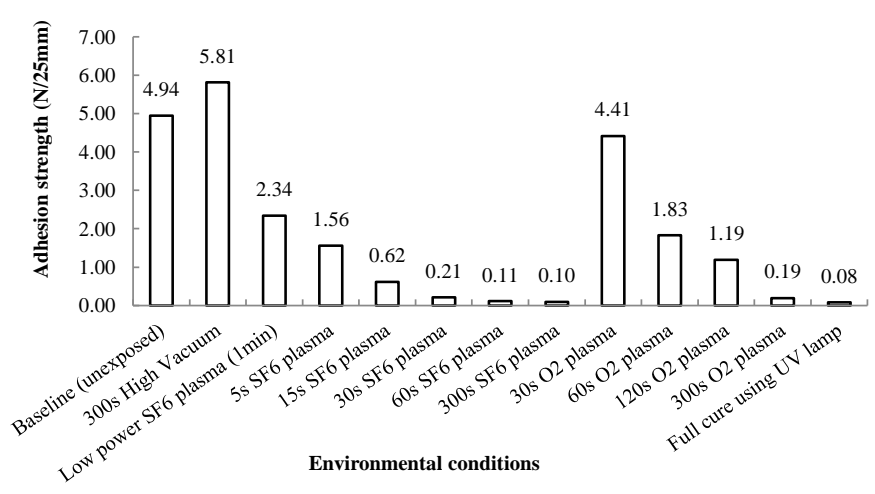

Fig. 11. $\mathrm{SF}_{6}$ and $\mathrm{O}_{2}$ Plasma exposure effect on PO10 dicing tape adhesion strength

An FTIR-ATR was employed as a more quantifiable method to measure changes in the adhesive layer chemistry through plasma induced adhesive curing.

If the correct amount of energy is absorbed by the photoinitiator present in the adhesive layer, homolytic cleavage can occur splitting the photoinitiator into very reactive free radicals. A localised cross-linking process then occurs as the reactive components of the photoinitiator targets the $\mathrm{C}=\mathrm{C}$ double bonds present in the $\mathrm{UV}$ resin, within the adhesive. The highly cross-linked polymer network entangles itself around the existing acrylic copolymer in the adhesive resulting in a reduction in adhesive mobility i.e. adhesive hardening and shrinking and hence a reduction in adhesive strength [21]. Measurement of this crosslinking process can 
be achieved by examining the level of $\mathrm{C}=\mathrm{C}$ bonds present within the adhesive layer as an indication of the extent of photoinitiated polymerization.

The absorption peak focused on was the acryloyl group $-\mathrm{H}_{2} \mathrm{C}=\mathrm{CH}-\mathrm{C}(=\mathrm{O})-\mathrm{R}$ (Fig 12).

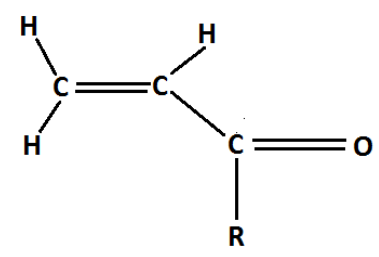

Fig. 12. The acryloyl functional group targeted during photoinitiated UV resin crosslinking

A sample of unexposed PO10 tape and another fully cured using a Lintec RAD-2000m/12 UV irradiation system were measured using the FTIR-ATR system. The maximum and minimum absorption peaks found at wave number $815 \mathrm{~cm}^{-1}$ were defined PreUV and Post-UV or $100 \%$ cross-linked. The samples measured after this were therefore a ratio of the absorbance measured on samples with the acryloyl group partially remaining versus the absorbance measured at $815 \mathrm{~cm}^{-1}$ on a sample of fully UV irradiated PO10. The UV cure ratio is represented in equation 1.

$$
\text { UV Cure Ratio }=\frac{(\text { absorbance of Pre UV sample }- \text { absorbance of Test sample })}{\text { absorbance of Pre UV sample }- \text { absorbance of post UV sample }} \times 100 \%
$$

Equation 1. UV Cure Ratio calculation

The $\mathrm{SF}_{6}$ plasma exposure FTIR-ATR results shown in Fig. 13 demonstrate that the presence of the acryloyl group is greatest in the unexposed PO10 samples. IR energy absorption at the wave number associated with the acryloyl group is directly dependent on exposure time in an $\mathrm{SF}_{6}$ plasma.

Samples were exposed to a high power (2000W, 35mTorr, 300sccm), SF 6 plasma for different times i.e. 5, 15, 30, 60 and 300s. Fig 13 shows that the UV Cure Ratios align well with the adhesion peel strength results thus proving that UV resin cross linking was being initiated through $\mathrm{SF}_{6}$ plasma exposure.

Results confirm that exposure to a high-power Oxygen plasma (2000W, 35mTorr, 300sccm) also induces UV curing of the adhesive albeit the rate is far slower than that seen on the samples exposed to $\mathrm{SF}_{6}$ plasma of the same power. 
FTIR-ATR Analysis of PO10 exposed to SF6 plasma

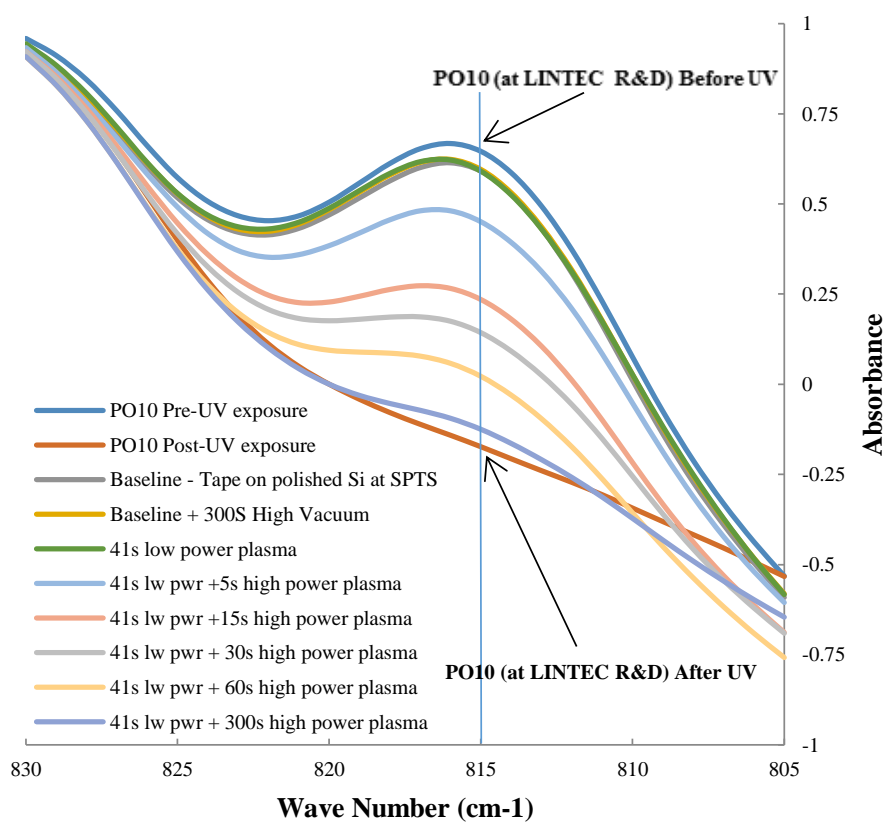

Fig. 13. FTIR-ATR Analysis of PO10 exposed to SF6 plasma

The $\mathrm{O}_{2}$ plasma exposure FTIR-ATR results also shown in Fig. 14 also show a similar trend to samples exposed to various lengths of $\mathrm{SF}_{6}$ plasma. However, UV energy absorption occurs at a slower rate.

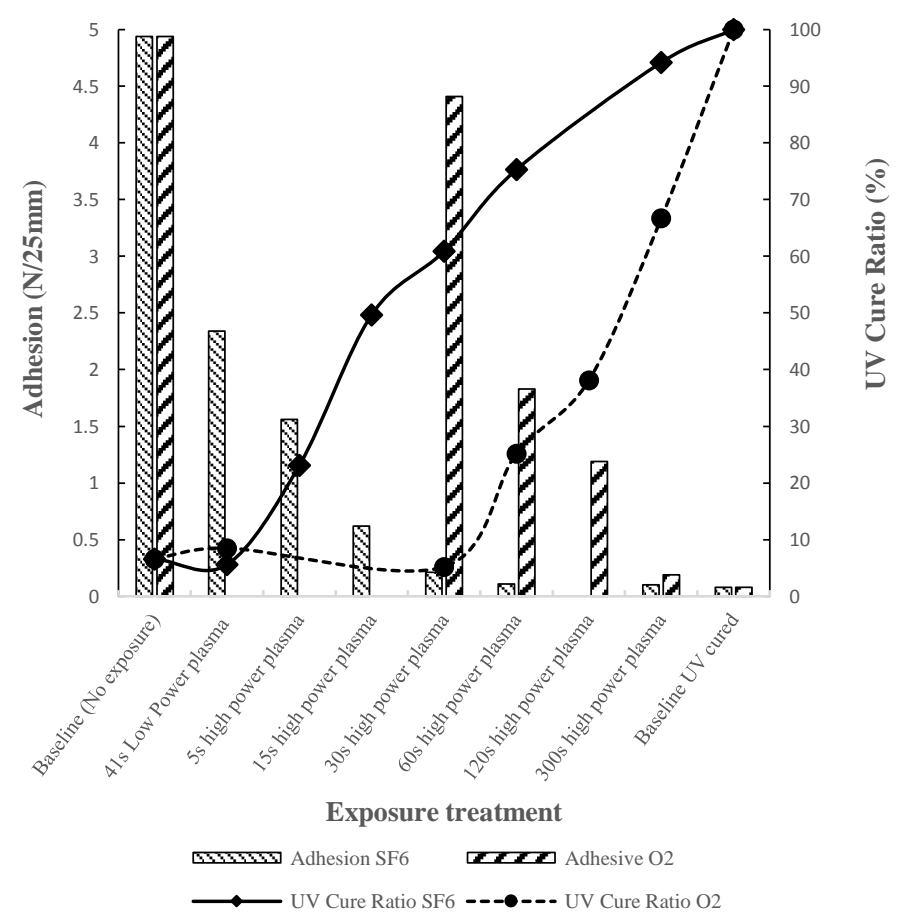

Fig. 14. Adhesive peel strength \& UV Cure Ratio results from samples exposed to differing intensities \& lengths of $\mathrm{SF}_{6}$ or $\mathrm{O}_{2}$ plasma 
An $\mathrm{SF}_{6}$ plasma spectra contains emission lines between 280 and $780 \mathrm{~nm}$. It is therefore reasonable to assume that the energy band required to cause photoinitiated polymerisation is present and the results we've seen with both adhesion peel strength and UV cure ratio measurements, demonstrate this.

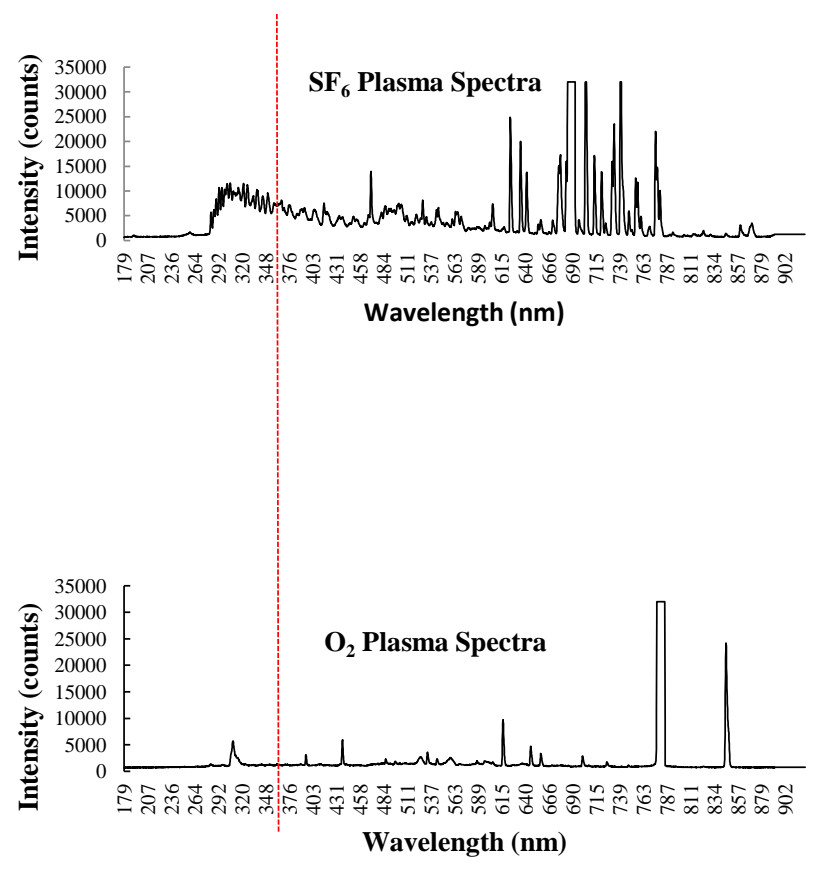

Fig. 15. $\mathrm{SF}_{6}$ and $\mathrm{O}_{2}$ plasma spectra as viewed with an OceanOptics USB4000 spectrometer. The red line highlights the UV wavelength of $365 \mathrm{~nm}$ targeted to trigger adhesive curing.

However, the emission spectrum of an oxygen plasma is very different, and a pure oxygen plasma does not include spectral lines around the $365 \mathrm{~nm}$ wavelength targeted for homolytic cleavage of the UV adhesives photoinitiator. See Fig. 15.

The OceanOptics USB4000 spectrometer used to analyze both $\mathrm{SF}_{6}$ and $\mathrm{O}_{2}$ plasma, uses a 3648 element $\mathrm{CCD}$ array detector with a range of 200 to $1100 \mathrm{~nm}$, so will not detect photonic energy with wavelengths less than 200nm [22]. Therefore, the OceanOptics spectrometer cannot provide a complete emission spectrum of all photonic energies generated within a plasma.

Specialized equipment is required to accurately measure Vacuum UV (VUV) radiation [23]. However, by using a combination of cut-off filters it is possible to identify the wavelength range responsible for inducing photo-initiated adhesive curing [24].

This was achieved by mounting the filters on the surface of the sample tape (see Fig. 16), exposing the samples to 300s of a high powered $\mathrm{SF}_{6}$ or $\mathrm{O}_{2}$ plasma and then measuring changes in the adhesive peel strength as previously described. 


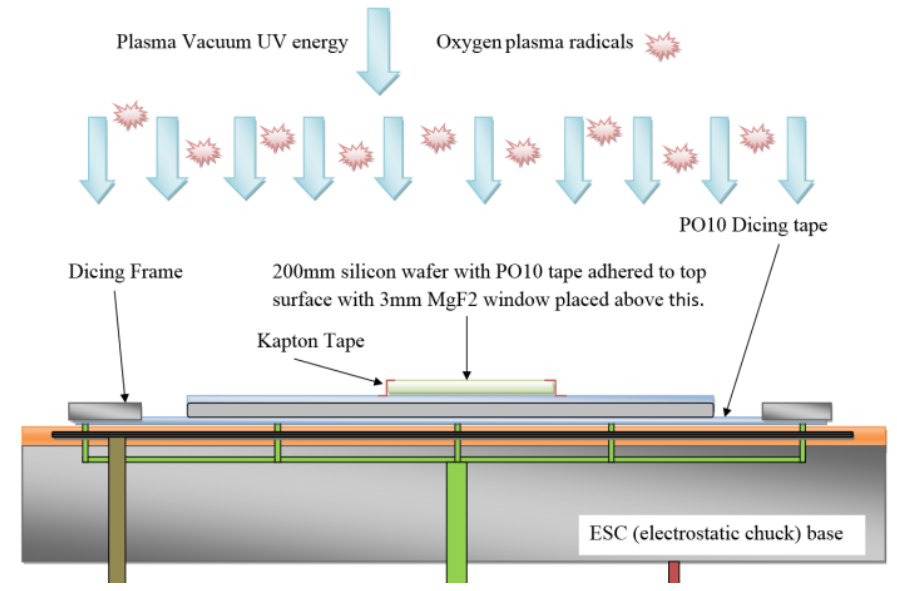

Fig. 16. Tape on polished silicon, then remounted on taped frame to provide a means of exposing the test tape to plasma. $\mathrm{MgF}_{2}$ filter placed in center of tape sample.

The results of the cut-off filter tests are shown in Table 3. The sample exposed to an $\mathrm{SF}_{6}$ plasma with the sapphire cut-off filter was fully cured proving that adhesive cross-linking occurs from wavelengths greater than $170 \mathrm{~nm}$ in this plasma.

The sample exposed to an $\mathrm{O}_{2}$ plasma with the sapphire cut-off filter was not cured in the area covered by the filter, proving that wavelengths lower than $170 \mathrm{~nm}$ from an $\mathrm{O}_{2}$ plasma are triggering the adhesive curing process.

The sample exposed to an $\mathrm{O}_{2}$ plasma with a magnesium fluoride filter was $98 \%$ cured, therefore proving that adhesive curing occurs from wavelengths greater than $112 \mathrm{~nm}$ but less than $170 \mathrm{~nm}$ in an $\mathrm{O}_{2}$ plasma.

Table 3. PO10 adhesive peel strength on unexposed tape, after 5 mins of an $\mathrm{SF}_{6}$ plasma without the use of a cut-off filter and then with a MgF $\mathrm{M}_{2}$ filter. Adhesive peel strength on PO10 tape exposed to 5 mins of an $\mathrm{O}_{2}$ plasma without the use of a filter, then with a $\mathrm{MgF}_{2}$ filter and lastly with sapphire filter.

\begin{tabular}{|c|c|c|c|}
\hline & Test condition & $\begin{array}{l}\text { Adhesive peel } \\
\text { strength } \\
(\mathrm{mN} / 25 \mathrm{~mm})\end{array}$ & $\begin{array}{l}\text { Adhesive } \\
\text { condition }\end{array}$ \\
\hline & Unexposed tape & 5000 & $0 \%$ cured \\
\hline $5 \mathrm{~min} \mathrm{SF}_{6}$ & $\begin{array}{l}\text { No cut-off filter } \\
\text { Sapphire }(<170 \mathrm{~nm} \\
\text { blocked })\end{array}$ & $\begin{array}{l}80 \\
80\end{array}$ & $\begin{array}{l}\text { Fully cured } \\
\text { Fully cured }\end{array}$ \\
\hline $5 \min \mathrm{O}_{2}$ & $\begin{array}{l}\text { No cut-off filter } \\
\mathrm{MgF}_{2}(<112 \mathrm{~nm} \\
\text { blocked }) \\
\text { Sapphire }(<170 \mathrm{~nm} \\
\text { blocked })\end{array}$ & $\begin{array}{l}150 \\
150\end{array}$ & $\begin{array}{l}98 \% \text { cured } \\
98 \% \text { cured }\end{array}$ \\
\hline
\end{tabular}


Although the design of a Rapier $200 \mathrm{~S}$ is tailored to provide a uniform etch rate across the wafer, the reality is that device singulation does not occur simultaneously i.e. the dicing lanes clear a little earlier at the intersections. To ensure the entire wafer has been singulated successfully, there is an over-etch step added that starts whenever process endpoint has been detected. The over-etch time is usually calculated as a percentage of the time taken to detect endpoint.

There was some concern that this over-etch portion of the plasma dicing process was contributing to a decrease in pre-UV adhesion strength of the dicing tape and at the same time causing an increase in post-UV adhesion strength. As the dicing lane clears and exposes the dicing tape, the UV energy from the plasma would irradiate the adhesive and induce photoinitiated crosslinking or curing, Fig. 17. This would not present a problem unless more than just the exposed adhesive is being cured. i.e. an encroachment under the singulated die.

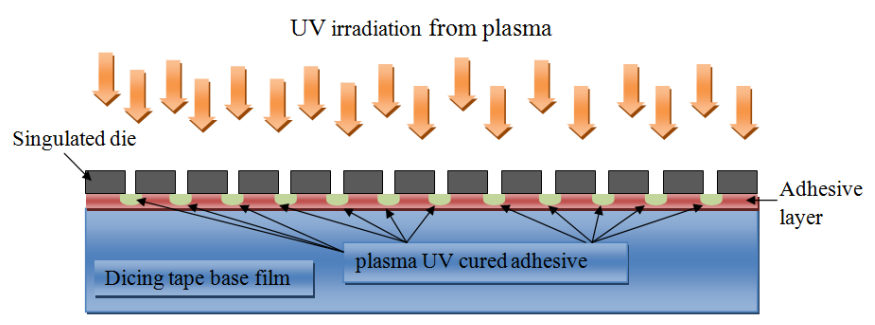

Fig. 17. Plasma exposure of adhesive as device singulation occurs

Test wafers $200 \mu \mathrm{m}$ thick and die size of $0.2 \times 0.2 \mathrm{~mm}$, [10 $\mu \mathrm{m}$ lanes, open area 9.3\%] were mounted on PO10 tape and etched using a standard plasma dicing recipe ('STD_Dicing_200_HR) until singulation was achieved. Samples were then mounted die-down on a silicon substrate using double-sided tape to provide the structural integrity for the diced wafer. The 50mm wide strips of double-sided tape were oriented at $45^{\circ}$ to the direction of the dicing lanes. This was done to prevent a "saw" shape on the load vs. displacement curve caused by the dicing lanes. Each diced wafer (with the supporting substrate) was then split into two halves. This allowed the dicing tape to be removed from one half and the other half to be swapped for fresh tape to compare adhesion strength of exposed tape versus that of fresh tape

The dicing tape was pre-cut in $25 \mathrm{~mm}$ wide strips at $10 \mathrm{~mm}$ spacings. The $10 \mathrm{~mm}$ strips of redundant tape were removed by hand to eliminate any interaction during the peel strengths tests.

The $180^{\circ}$ adhesive peel strength was measured using a speed of $300 \mathrm{~mm} / \mathrm{min}$ in the up direction at a data sampling rate of 40 samples per second. The set-up is shown in Fig. 18a. The measured load was averaged between $20 \mathrm{~mm}$ and $70 \mathrm{~mm}$ displacement. Each data point was an average of results from 4 separate tape strips on a given substrate. Adhesive strength measurements were taken on singulated samples pre-UV released and on separate samples post-UV release (on both original tape and fresh tape).

Note that there was there was a delay between processing and measurement of approximately 14 days. An example of a load versus displacement curve is shown in figure $18 \mathrm{~b}$. The average load measured between $20 \mathrm{~mm}$ and $70 \mathrm{~mm}$ was $1.76 \mathrm{~N}$. This equates to an average adhesive peel strength of $1760 \mathrm{mN} / 25 \mathrm{~mm}$. 


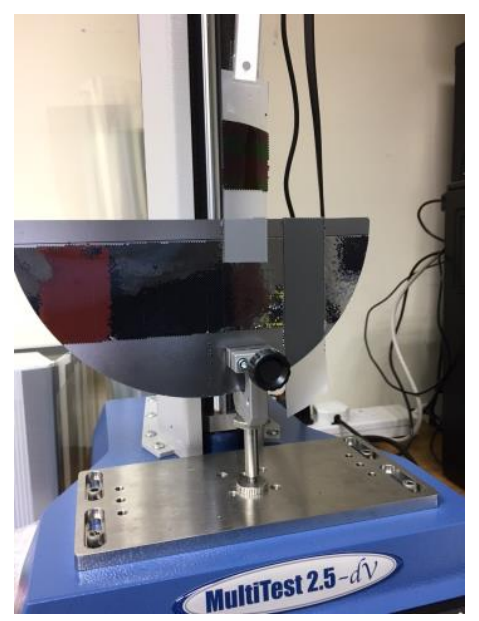

Fig. 18a. Adhesive peel strength measurement on $25 \mathrm{~mm}$ strips of dicing tape attached to singulated devices

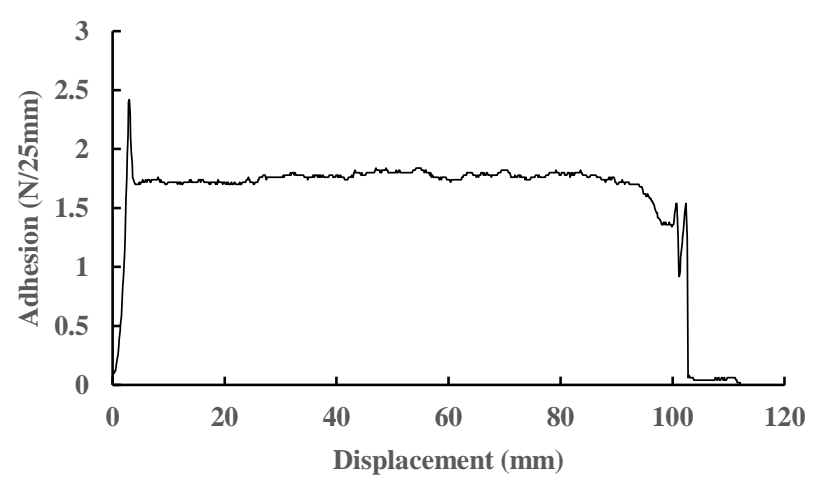

Fig 18b. The load v displacement curve of original PO10 tape holding small die, measured 14days after plasma dicing.

The pre-UV adhesive peel strength results from the original tape used during the full plasma dicing process were much lower than anticipated for the $9.3 \%$ open area test sample. The sample remounted on fresh tape measured markedly higher proving that the dicing tape adhesive strength is being altered during or at the end of the dicing process. This is shown in Fig. 18c. It should be noted that in previous tests of the same nature, the pre-UV adhesive strength on the original tape samples varied with higher adhesion strength measured as the delay between processing and measurement increased.

Post-UV adhesive peel strength was low at $270 \mathrm{mN} / 25 \mathrm{~mm}$, but lower still when samples were transferred to fresh tape and then UV irradiated $(100 \mathrm{mN} / 25 \mathrm{~mm})$. 


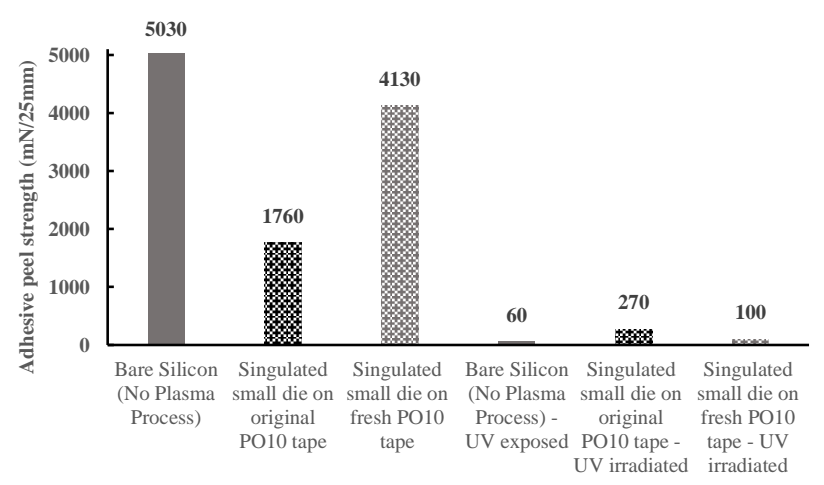

Fig. 18c. Adhesive peel strength of PO10 tape $-200 \mu 2,200 \mu$ thick die with $10 \mu$ dicing lanes (plasma dice $->$ measurement $=14$ days)

Worth noting is that all samples were tape mounted and plasma diced to singulation on the same day and the samples to be mounted on fresh tape were transferred immediately after the dicing process. All samples were shipped to Munich for UV exposure on a LINTEC RAD 2010 and then returned to the UK for measurement. This was part of a larger investigation to determine how much of an effect an air purge versus $\mathrm{N}_{2}$ purge has on UV exposure and adhesive cross-linking.

There were some delays incurred during this investigation and in order to understand the effect of these delays, several more samples were prepared. The first set were UV exposed prior to plasma dicing and the second were UV exposed after the dicing process. Adhesive peel strength was measured after 1 hour on half of each sample set and then again after 7 days. The results as shown in Fig. 18d showed that adhesion increases the longer the delay between processing and measurement, but more importantly there was no significant difference between the adhesion strength of samples where the adhesive had been cured prior to plasma dicing and those UV irradiated after dicing. This is encouraging in that it shows that adhesive strength can still be minimized through correct UV exposure (from the back side of tape) to ensure that die pick is successful and damage free.

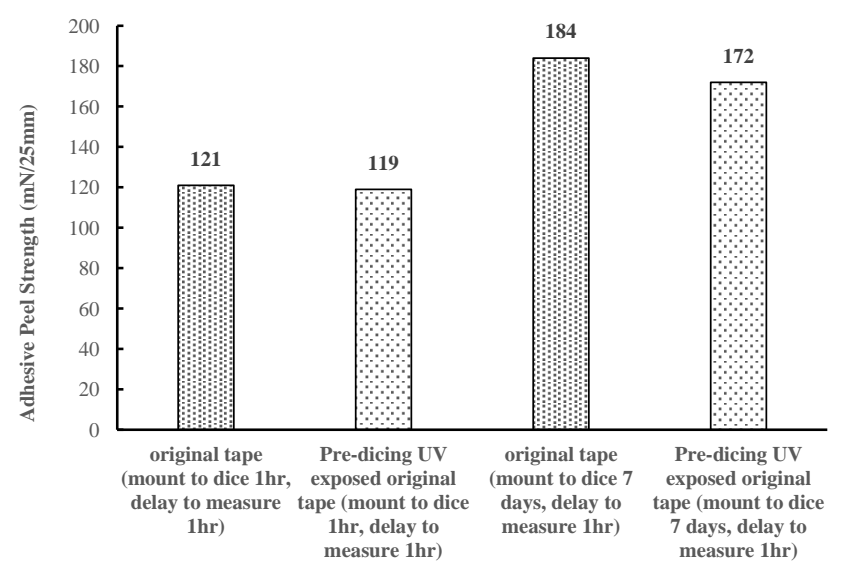

Fig. 18d. Adhesive peel strength of PO10 tape $-200 \times 200 \mu \mathrm{m}, 200 \mu \mathrm{m}$ thick die with $10 \mu \mathrm{m}$ dicing lanes (varying delays between plasma dice and measurement)

In order to limit die stresses induced during the die pick-up process, low adhesive strength is essential [25-27]. The results from these last two adhesive peel strength tests on singulated material show that to minimize post UV adhesive strength, the delay between tape mount to plasma dicing should be as short as possible as should the delay between plasma dice and die Pick-Up. Most Tape manufacturers call for these delays to be less than 5 days. 
Similar adhesive peel strength tests on singulated device of the same sized die were completed for the non-UV tape PO6 and the higher adhesive strength polyolefin tape PO2. The results on both sets of tests were very close between the original tape and samples remounted on fresh tape. (PO6 - 1145mN/25mm versus 1008mN/25mm, PO2 - 1919mN/25mm versus 1947mN/25mm).

As a furtherance to the adhesion strength measurements after substrate singulation tests, an additional set of experiments designed to measure the amount of force required to separate singulated die from the supporting tape after either saw dice or plasma dice singulation was completed at LINTEC R\&D in Japan. The details and results of these tests were reported in the conference paper 'Dicing Tape Performance in a Plasma Dicing Environment' [17]. It was shown that pick-up force measured on PO10 tape from plasma diced dies are comparable with the same sized dies singulated using a saw dicing system.

\section{CONCLUSION}

Results from the 'robustness tests' on over 50 dicing tapes suggest that Polyolefin UV tapes with a low to medium adhesive strength, most often have the lowest out-gassing rate and are least likely to declamp during a plasma dicing process. However, there are exceptions and further analysis of tape outgassing behavior, through the use of Gas Chromatography-Mass Spectrometry would provide a greater understanding and aid in advancing this research.

Indirect plasma exposure (through tape) of the adhesive layer can initiate adhesive curing at different rates dependent on plasma species. Although there is evidence that partial curing of the adhesive may be occurring around the edge of singulated die seen as a difference in pre-UV adhesive peel strength on original versus freshly mounted sample, there is no detrimental effect on the postUV adhesive peel strength. That is, the adhesive is still able to be fully cured. This supports the findings in a previous set of experiments to measure die pick-up force where it was shown that on medium to low adhesion PO tape the pick-up force on plasma diced dies is comparable with the same size dies that had been saw diced.

\section{ACKNOWLEDGMENT}

H. Fulton would like to acknowledge the assistance of Matt Day (SPTS Etch Applications team) and Martin Hanicinec (former member of SPTS Etch Applications Group, now studying for his $\mathrm{PhD}$ at University College London), Naoya Saiki and Kosei Fukumoto (from the Electronics Materials Lab, LINTEC Corporation Research Centre in Japan). In addition, many thanks to Edwin Thys of Nitto Denko Corporation, Michael Grethe of Furukawa Electric Group, Brian Raeburn of Disco Hi-Tec UK Ltd (for tapes from Denka Company Limited), Peter Summers of SPS Europe (for tapes from Ultron Systems Inc), Shunsuki Inazuka of D\&X Co. Ltd., and Shingo Esaki of Sekisui Chemical Co. Ltd. for arranging the supply of various dicing tapes to be tested.

\section{REFERENCES}


[2] F. Wei, T. Tabuchi, T. Lazerand, C. Johnston, K. Mackenzie, and M. Notarianni, "Plasma Dicing Fully Integrated Process-Flows Suitable for BEOL Advanced Packaging Fabrications," in 2017 IEEE 67th Electronic Components and Technology Conference (ECTC), 2017, pp. 350-357.

C. Johnston and F. Piallat, "Defect-Free Dicing for Higher Device Reliability," in 2018 7th Electronic System-Integration Technology Conference (ESTC), 2018, pp. 1-4.

[4] D. Lishan et al., "Wafer dicing using dry etching on standard tapes and frames," in International Symposium on Microelectronics, 2014, vol. 2014, no. 1, pp. 000148-000154: International Microelectronics Assembly and Packaging Society.

[5] R. Barnett, "Plasma Dicing 300mm Framed Wafers \&\#x2014; Analysis of Improvement in Die Strength and Cost Benefits for Thin Die Singulation," in 2017 IEEE 67th Electronic Components and Technology Conference (ECTC), 2017, pp. 343-349.

[6] K. TAKAHASHI, "Requirements and Development Trend of Dicing Tapes," NIPPON GOMU KYOKAISHI, vol. 85, no. 2, pp. 52-57, 2012.

[7] T. K. S. Takyu, "Chip Thinning Technologies for Chip Stacking Packages," presented at the ICEP 2013, Osaka, Japan, April $2013,2013$.

[8] N. Saiki, K. Inaba, K. Kishimoto, H. Seno, and K. Ebe, "Study on Peeling Behavior in Pick-up Process of IC Chip with Adhesive Tapes," Journal of Solid Mechanics and Materials Engineering, vol. 4, no. 7, pp. 1051-1060, 2010.

[9] S. Takyu et al., "A Novel Dicing Technologies for WLCSP Using Stealth Dicing through Dicing Tape and Back Side Protection-Film," in 2016 IEEE 66th Electronic Components and Technology Conference (ECTC), 2016, pp. 1241-1246.

[10] Laermer, Franz, Schilp, and Andrea, "Method of anisotropically etching silicon," Patent 5501893, 1996.

[11] J. F. Daviet, L. Peccoud, and F. Mondon, "Electrostatic Clamping Applied to Semiconductor Plasma Processing: I. Theoretical Modeling," Journal of the Electrochemical Society, vol. 140, no. 11, pp. 3245-3246, 1993.

[12] B. Wu, A. Kumar, and S. Pamarthy, "High aspect ratio silicon etch: A review," Journal of applied physics, vol. 108, no. 5, p. 9 , 2010.

[13] D. Thomas, M. Muggeridge, J. Hopkins, N. Launay, H. Ashraf, and T. Barrass, "Deep Silicon Etching-Increasingly Relevant> 20 Years on!," ECS Transactions, vol. 72, no. 19, pp. 9-22, 2016.

[14] Z. JIS, "0237: 2009 Testing methods of pressure-sensitive adhesive tapes and sheets," ed, 2016.

[15] BS EN ISO 29862:2019 - Self adhesive tapes. Determination of peel adhesion properties, 2019.

[16] BS EN ISO 29864:2019 - Self adhesive tapes. Measurement of breaking strength and elongation at break, 2019.

[17] S. Fulton, O. Ansell, J. Hopkins, T. Umemoto, and T. Nishida, "Dicing Tape Performance in a Plasma Dicing Environment," in 2018 IEEE 20th Electronics Packaging Technology Conference (EPTC), 2018, pp. 229-236.

[18] M. Patel et al., "Volatile out gassing characteristics of highly filled ethylene vinyl acetate binder materials: Gas phase infra-red spectroscopy," Polymer Testing, vol. 32, no. 2, pp. 313-320, 2013/04/01/ 2013.

[19] L. Mauri, E. Rizzi, M. Moraja, and M. Campaniello, "The discrete vacuum packaging reliability issue in MEMS," in 2013 Eurpoean Microelectronics Packaging Conference (EMPC), 2013, pp. 1-4.

[20] R. Barnett, O. Ansell, M. Hanicenic, and J. Hopkins, "Novel end-point solution for improvement in die strength and yields with plasma dicing after grind in volume production," in 2017 IEEE 19th Electronics Packaging Technology Conference (EPTC), 2017, pp. 1-4.

[21] D. Ghim and J. H. Kim, "Effects of composition and layer thickness of a butyl acrylate/acrylic acid copolymer on the adhesion properties," Korean Journal of Chemical Engineering, vol. 33, no. 2, pp. 707-710, 2016/02/01 2016.

[22] C. J. Pugh, "End Point Detection in Reactive Ion Etching," PhD, Electrical and Electronic Engineering, (UCL) University College London, Section 2.7 p26-27, 2013.

[23] E. J. Iglesias, F. Mitschker, M. Fiebrandt, N. Bibinov, and P. Awakowicz, "in situ measurement of vuv/uv radiation from low-pressure microwaveproduced plasma in ar/o 2 gas mixtures," Measurement Science and Technology, vol. 28, no. 8, p. 085501, 2017.

[24] G. W. Drake, Springer handbook of atomic, molecular, and optical physics. Springer Science \& Business Media, 2006.

[25] K. Ebe, H. Seno, and K. Horigome, "UV curable pressure-sensitive adhesives for fabricating semiconductors. I. Development of easily peelable dicing tapes," Journal of Applied Polymer Science, vol. 90, no. 2, pp. 436-441, 2003.

[26] K. Horigome, K. Ebe, and S. i. Kuroda, "UV curable pressure - sensitive adhesives for fabricating semiconductors. II. The effect of functionality of acrylate monomers on the adhesive properties," Journal of Applied Polymer Science, vol. 93, no. 6, pp. 2889-2895, 2004.

[27] S.-W. Lee, J.-W. Park, H.-J. Kim, K.-M. Kim, H.-I. Kim, and J.-M. Ryu, "Adhesion Performance and Microscope Morphology of UV-Curable Semiinterpenetrated Dicing Acrylic PSAs in Si-Wafer Manufacture Process for MCP," Journal of Adhesion Science and Technology, vol. 26, no. 1-3, pp. 317-329, 2012/01/01 2012. 


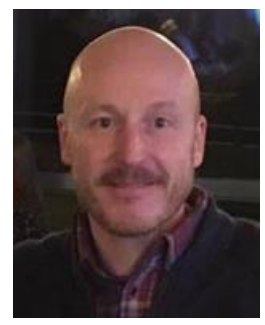

Harry Fulton received a Higher National Certificate in electronic and electrical engineering from The James Watt College, Greenock, Scotland in 1989 and a National Certificate in Natural Sciences at the Open University in 2003. He is currently pursuing a Ph.D. degree in electronic engineering with Swansea University. He has worked as an equipment engineer in the semiconductor industry since 1984, joining SPTS Technologies Ltd., Newport, in 2007. His research interests are in Plasma Dicing of semiconductor devices.

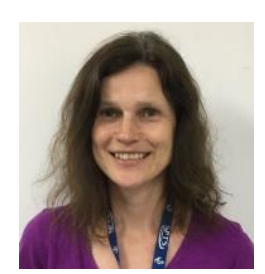

Janet Hopkins studied Physics and Chemistry of Materials at the University of Durham followed by a PhD in plasma surface modification. She joined SPTS Technologies in 1995 as a process engineer in the Si etch group. During her career she has worked on developing different plasma sources and processes and is the author of many papers and patents. She is currently the Etch Applications manager, currently focusing on plasma dicing.

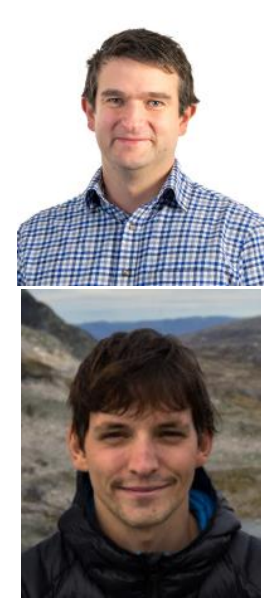

Oliver Ansell studied Electrical and Electronics Engineering at the University of Bristol followed by a $\mathrm{PhD}$ in optoelectronic communications. He joined SPTS Technologies in 2007 as a process engineer and has released various patents and papers. He is now an Etch Technologist working in the Etch Applications group focusing on plasma dicing and endpoint solutions.

Martin Hanicinec received his master's degree in plasma physics at Masaryk University, Brno, Czechia. He worked for SPTS Technologies between 2015 and 2018 as an Etch Applications process engineer participating on the development of the plasma dicing technology. He is currently pursuing a $\mathrm{PhD}$. in plasma modelling at University College London. His research interests are plasma kinetic models and chemical reduction techniques.

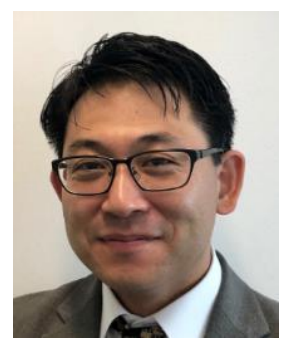

Takuo Nishida has graduated the master's program at the University of Gunma graduate school of applied chemistry in 2005. He entered LINTEC Corporation in 2005 and engaged in the development of release materials and adhesive materials for labels. He has worked as a tape chemist in the semiconductor industry since 2012, transferring LINTEC Advanced Technologies (Europe) GmbH as a tape application engineer in 2016. His research interests are new dicing process and suitable tapes for this process.

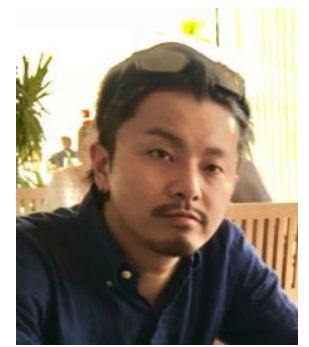

Dicing process.
Taku Umemoto received a bachelor's degree of intercultural communication from Hosei University, Tokyo, Japan in 2008. Since 2014 he has worked as a sales rep in LINTEC Advanced Technologies (Europe) GmbH, Munich, Germany. His current interests are in incorporating semiconductor-related adhesive tapes and equipment for Plasma

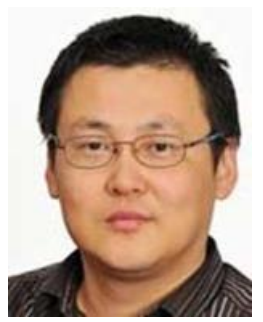

Lijie $\mathrm{Li}$ is a professor at the College of Engineering, Swansea University, United Kingdom. His research interests are in developing MEMS transducers, energy harvesting devices, semiconductors devices, piezotronics, optical and radio frequency (RF) MEMS devices and systems. Moreover, he is interested in first principle study of nanodevices and nanomaterials, as well as machine learning techniques with applications in transducers. $\mathrm{He}$ is Fellow of IET, and senior member of IEEE. 